\title{
Laudatio für Prof. Dr. med. Wolfgang Queißer
}

Prof. Dr. med. Wolfgang Queißer steht wie kaum an anderer in Deutschland für Internistische Onkologie und für patientenorientierte klinische Forschung. In den 30 Jahren seiner Tätigkeit am Universitätsklinikum Mannheim der Universität Heidelberg hat er maßgeblich zur Entwicklung und Etablierung des Faches Internistische Onkologie beigetragen. Vor Ort geschah dies durch die Schaffung einer integrierten ambulanten und stationären Einheit für die interdisziplinäre Behandlung von Tumorpatienten, des Onkologischen Zentrums, sowie durch die Gründung eines Onkologischen Arbeitskreises am Mannheimer Klinikum. Beide Gründungen haben die Jahre und Jahrzehnte überdauert und sind richtungsweisend geworden für die Entwicklung der Internistischen Onkologie in Deutschland.

Wie viele Internistische Onkologen kommt Wolfgang Queißer aus der Hämatologie und ist zeit seines Lebens zugleich Hämatologe und Internistischer Onkologe geblieben. Wolfgang Queißer hat aus seiner Erfahrung mit der Therapie zuvor unheilbarer Leukämien und Lymphome die Notwendigkeit abgeleitet, diese Therapieerfolge auf die soliden Tumoren zu übertragen. Die Logik und Notwendigkeit des Vorgehens leiteten sich aus der Häufigkeit der soliden Tumoren, ihrem meist unerbittlichen Verlauf und der menschlich-ärztlichen Dringlichkeit therapeutischer Fortschritte ab. Wolfgang Queißer ist damit ein Vorkämpfer für Fortschritte bei der Therapie solider Tumoren und für ein besseres Management der von diesen Tumoren betroffenen Patientengruppe geworden. Sein Eintreten hierfür und für eine Etablierung und Weiterentwicklung der Onkologie haben ihm nicht nur den Dank seiner Patienten, sondern auch ein hohes nationales und internationales Ansehen unter Fachkollegen eingebracht.

Wolfgang Queißer wurde am 16. Mai 1936 in Pethau in jenem kleinen Zipfel von Sachsen geboren, der östlich der Lausitzer Neiße liegt und nach dem Krieg an Polen abgetreten werden musste. Nach der Ausweisung lebte die Familie zunächst in Zittau. Von 1950 bis 1957 besuchte Wolfgang Queißer das Gymnasium in Plön in Schleswig-Holstein. Es folgte das Me- dizinstudium in Berlin, Freiburg i.Br. und Heidelberg, wo er 1963 das Staatsexamen ablegte. Dank seiner sehr guten Leistungen wurde er Stipendiat der Studienstiftung des Deutschen Volkes. Nach der Medizinalassistentenzeit in Ludwigshafen, Rendsburg und Freiburg i.Br., wo er über ein biochemisches Thema promovierte, folgte die Tätigkeit am Pathologischen Institut in Kiel bei Professor Karl Lennert. Hier erwarb er die Grundlagen für seine späteren Arbeiten zum Knochenmark. 1967 wechselte er zum damals neu gegründeten Zentrum für Innere Medizin der Universität Ulm zu Professor Hermann Heimpel. In Ulm erwarb er seinen Facharzt für Innere Medizin und habilitierte sich 1972 über «Die Anwendung zytophotometrisch-autoradiographischer Methoden für die Untersuchung der Proliferation normaler und gestörter hämopoetischer Zellsysteme». 1971 wurde er nach Mannheim berufen. 1973 erfolgte die Gründung des interdisziplinären Onkologischen Arbeitskreises. 1976 gründete er mit Kollegen die Arbeitsgemeinschaft Internistische Onkologie (AIO) der Deutschen Krebsgesellschaft sowie im gleichen Jahr die Chemotherapiegruppe Gastrointestinaler Tumoren (CGT) in Mannheim. 1977 wurde das Onkologische Zentrum gegründet und Wolfgang Queißer zum leitenden Arzt ernannt. 1978 erfolgte die Herausgabe des Buches «Das Knochenmark: Morphologie, Funktion, Diagnostik» (Stuttgart, Thieme), das noch heute zu den Standardwerken auf diesem Gebiet zählt. 1979 wurde er Mitglied des Lenkungsausschusses des Tumorzentrums Heidelberg/Mannheim, und 1980 erfolgte die Ernennung zum C2-Professor für Internistische Onkologie auf Dauer. Er war 1984 Mitbegründer der Oberrheinischen Arbeitsgemeinschaft für Onkologie (OAO). Von 1984 bis 1992 war er Vorsitzender der Phase-II-Studiengruppe der AIO und Mitglied des Vorstands und von 1986 bis 1989 Vorsitzender der Süddeutschen Hämoblastosegruppe (SHG). Seit 1989 ist er federführender Schriftleiter der Zeitschrift OnKOLOGIE. Er ist Autor bzw. Koautor von 453 Publikationen, hat 5 Bücher herausgegeben, 52 Promotionen betreut und 2 seiner Mitarbeiter zur Habilitation geführt.

\begin{tabular}{ll}
\hline KARGER & ( ) 2001 S. Karger GmbH, Freiburg \\
$\begin{array}{l}\text { Fax +49761 4520714 } \\
\begin{array}{l}\text { E-mail Information@Karger.de } \\
\text { www.karger.com }\end{array}\end{array}$ & Accessible online at: \\
www.karger.com/journals/onk
\end{tabular}


Was macht den Erfolg der Persönlichkeit Wolfgang Queißers aus?

Hier sind vor allem zu nennen der Blick für das Wesentliche sowie eine große Ausdauer und Beharrlichkeit. Hinzu kommen seine Fähigkeit zur Kooperation, ohne dabei seine einmal für richtig erkannten Ziele aus den Augen zu verlieren, und seine besondere Fähigkeit, frühzeitig Entwicklungen zu erkennen und in Organisationsstrukturen umzusetzen. Dies hat sich insbesondere bei der Gründung des Onkologischen Arbeitskreises und des Onkologischen Zentrums in Mannheim gezeigt. Ebenso hat er frühzeitig erkannt, dass sich eine zukunftsfähige Onkologie nur durch überregionale Kooperation und daraus resultierende klinische Forschung unter Einschluss der pharmazeutischen Industrie erreichen lässt. Er gehörte zu den ersten, die in Deutschland das Dilemma der klinisch-onkologischen Forschung aktiv zu überwinden suchten. So etablierte er in den frühen siebziger Jahren erste multizentrische Therapiestudien bei soliden Tumoren, setzte sich mit den Grundlagen der klinischen Prüfung auseinander und gründete 1976 die bereits erwähnte CGT, die sich fortan mit zahlreichen Studien zur palliativen und adjuvanten Chemotherapie von Magen-Darm-Tumoren befasste.

Das Bild der Persönlichkeit Wolfgang Queißers wird durch seine künstlerischen Neigungen abgerundet. Das Onkologische Zentrum in Mannheim war zugleich auch Ausstellungsort für künstlerische Photographien, die er auf Reisen, aber auch in Mannheim anfertigte und ausstellte.

Nach Beendigung seiner Hochschultätigkeit bleibt sich Wolfgang Queißer in seinen wesentlichen Eigenschaften treu: Er hat frühzeitig die zukünftige Bedeutung der präventiven Onkologie erkannt und wird ab Oktober in Mannheim eine Praxis für präventive Onkologie begründen. Sicher wird er auch damit Erfolg haben.

Wir wünschen ihm für seinen weiteren privaten und beruflichen Weg alles Gute.

R. Hehlmann, Mannheim 